1-Methoxynaphthalin das Moment dann in Richtung der größten Molekülausdehnung, wenn die $\mathrm{CH}_{3}$. Gruppe sich mit ihrem Schwerpunkt in der Ringebene befindet. Davon ist aus sterischen Gründen nur eine Lage möglich. 2-Methoxynaphthalin dagegen besitzt in diesen Gruppenstellungen nur eine sehr kleine Momentkomponente in der großen Molekülachse. Die Momentrichtung ist also bei den Methoxy-Verbindungen gerade um etwa $90^{\circ}$ gegen die bei den Bromnaphthalinen verschoben. Die Abschätzung nach PERrin führt hier auf ein $\tau_{1}$-Verhältnis zwischen 1-und 2-Verbindung von 1,3, während 1,35 beobachtet ist. Wenn die Gruppe senkrecht zur Ringebene stehen würde, liefert die Perrinsche Theorie keinen Unterschied in den Relaxationszeiten.

Roberti und Sмyth ${ }^{12}$ schließen aus Messungen an der reinen Flüssigkeit auf eine große innere Beweglichkeit des 2-Methoxynaphthalin, was wir in der verdünnten Lösung nicht bestätigen können. Wegen ihres hohen Schmelzpunktes $\left(75^{\circ} \mathrm{C}\right)$ ist die reine Flüssigkeit bei $80^{\circ} \mathrm{C}$ gemessen, so daß vielleicht eine Parallele zum Verhalten des Hydrochinondimethyläthers zu ziehen ist.

Da die unterschiedlichen Relaxationszeiten der beiden Methoxynaphthaline dadurch eine Erklärung finden könnten, daß die $\mathrm{OCH}_{3}$-Gruppe sich hauptsächlich mit ihrem Schwerpunkt in der Ringebene aufhält, liegt es nahe, in dieser Einstellung eine Besonderheit zu vermuten. Dazu kommt die an den beiden Molekülen beobachtete Unbeweglichkeit der Gruppe, die ebenfalls durch eine fixierte Lage gegeben wäre. Die ebene Anordnung Ring - O - C ist nun gerade dadurch bevorzugt, daß sich in ihr am günstigsten als mesomerer Grenzzustand eine Doppelbindung zwischen dem Ring und dem Sauerstoffatom ausbilden kann, indem ein Elektronenpaar vom Sauerstoff in die Bindung wandert. Eine derartige Elektronenverteilung ist wiederum mit der Starrheit des Moleküls über Zeiten, die länger als die gemessene Relaxationszeit der Größenordnung $10^{-11} \mathrm{sec}$ sind, im Einklang. Dieses Bild kann man versuchsweise auf die anderen Molekeln übertragen, indem man postuliert, daß die Gruppe dann beweglich ist, wenn sie sich nicht in der Ringebene befindet. Dafür würde sprechen, daß Gruppen in $o$-Stellung sowohl beim Veratrol als auch beim Trimethylanisol, die ein Einschwingen in die Ringebene - mindestens einseitig - sterisch behindern, zu einer Erhöhung des sich schnell orientierenden Momentanteils führen.

An den Messungen beteiligten sich Frau F. Hanna, Frau U. Arnemann und Herr P. KNobloch, wofür ihnen auch hier vielmals gedankt sei. Ebenso schulden wir der Deutschen Forschungsgemeinschaft unseren besten Dank, da sie durch Sachbeihilfen die Arbeiten unterstützte.

\title{
Zustandsfunktionen für die Atome Lithium bis Neon
}

\author{
Von B. KockeL \\ Aus der Forschungsgruppe Theoretische Chemie der Deutschen Akademie der Wissenschaften zu Berlin \\ und dem Institut für Theoretische Physik der Universität Gießen \\ (Z. Naturforschg. 16 a, 1021-1026 [1961] ; eingegangen am 15. Juli 1961) \\ Für die Atome $\mathrm{Li}$ bis $\mathrm{Ne}$ und die positiven Ionen $\mathrm{Li}^{+}$bis $\mathrm{Ne}^{+}$werden $\mathrm{Zustandsfunktionen} \mathrm{vom}$ \\ SLATER-Typ angegeben, die nur zwei durch Variation bestimmte Konstanten enthalten, je eine für die \\ K- und L-Schale. Zustandsfunktionen für negative Ionen werden diskutiert.
}

\section{Zustandsfunktionen für die neutralen Atome Lithium bis Neon}

Für die Atome Lithium bis Neon und alle ihre positiven Ionen haben Morse, Young und Haurwitz ${ }^{1}$ sowie Duncanson und Coulson ${ }^{2}$ und Tubis ${ }^{3} \mathrm{Zu}$ standsfunktionen für die tiefsten Zustände mit Hilfe

1 P. M. Morse, L. A. Young u. E. S. Haurwitz, Phys. Rev. 48, 948 [1935]. des Ansatzes I der Tab. 1 (s. u.) bestimmt. Für Molekülberechnungen ist es von Vorteil, wenn man noch einfachere, also weniger als die vier Konstanten $k, k \alpha, k \beta, k \gamma$ des Ansatzes I enthaltende Funktionen verwendet. Eine solche Vereinfachung ist zunächst möglich, wenn man die Konstante $k \alpha$ einspart. So entsteht der Ansatz II. Er unterscheidet sich vom

\footnotetext{
2 W. E. Duncanson u. C. A. Coulson, Proc. Roy. Soc., Edinburgh 62, 37 [1942].

3 A. Tubis, Phys. Rev. 102, 1049 [1956].
} 
Ansatz I dadurch, daß I den 2s-Funktionen freistellt, wie sie die Orthogonalität zu den ls-Funktionen erreichen, während Ansatz II vorschreibt, daß dazu die 1s-Funktion selbst mitverwendet wird. Darüber hinaus ist es auch noch möglich, die Exponenten für die 2s- und 2p-Funktionen gleich zu wählen, was zu dem nur 2-konstantigen Ansatz III führt.

\begin{tabular}{|l|c|c|c|}
\hline Ansatz & $1 \mathrm{~s}$ & $2 \mathrm{~s}$ & $2 \mathrm{p}$ \\
\hline I & $e^{-k r}$ & $\left\lfloor r e^{-k \beta r}, e^{-k \alpha r}\right\rfloor$ & $r e^{-k \gamma r} \mathfrak{y}_{1}^{m}(\vartheta, \varphi)$ \\
II & $e^{-k r}$ & $\left\lfloor r e^{-k \beta r}, e^{-k r}\right\rfloor$ & $r e^{-k \gamma r} \mathfrak{y}_{1}^{m}(\vartheta, \varphi)$ \\
III & $e^{-k r}$ & $\left\lfloor r e^{-k \beta r}, e^{-k r}\right\rfloor$ & $r e^{-k \beta r} \mathfrak{y}_{1}^{m}(\vartheta, \varphi)$ \\
\hline
\end{tabular}

Tab. 1. Drei mögliche Ansätze für $1 \mathrm{~s}-, 2 \mathrm{~s}$ - und 2 p-Funktionen. $\lfloor\ldots, \ldots\rfloor$ heißt Linearkombination der beiden durch Komma getrennten Funktionen. Die zur Normierung und zur Orthogonalisierung der $2 \mathrm{~s}-\mathrm{zu}$ den $1 \mathrm{~s}$-Funktionen nötigen Faktoren sind nicht mit eingetragen.

Für den Ansatz I findet man die Konstanten- und Energiewerte in den zitierten Arbeiten. Für den Ansatz II wurden sie von Kockel und RühlemanN ${ }^{4}$ und RansiL ${ }^{5}$ angegeben. In den Tab. 2 und 3 sind sie zum Vergleich und mit Ergänzung der Werte $k \beta$ und $k \gamma$ für die Arbeit ${ }^{4}$ wiederholt. Die Abweichungen sind bis auf eine Ausnahme gering. Sie erklären sich daraus, daß Kockel und Rühlemann ein Interpolationsverfahren zwischen den Energiewerten für $\beta$ und $\gamma=0,22 ; 0,24 ; \ldots ; 0,32$ anwandten. Bei der Ausnahme, dem $k \beta$-Wert für Bor, ist jedoch ein Druck- oder Rechenfehler in einer der Arbeiten zu vermuten.

\begin{tabular}{|l|l|l|l|l|}
\hline & & $k$ & $k \beta$ & $k \gamma$ \\
\hline $\mathrm{Li}$ & ${ }^{2} \mathrm{~S}$ & 2,6865 & 0,6372 & - \\
$\mathrm{Be}$ & ${ }^{1} \mathrm{~S}$ & 3,6847 & 0,9562 & - \\
$\mathrm{B}$ & ${ }^{2} \mathrm{P}$ & 4,6794 & 1,33825 & 1,21055 \\
$\mathrm{C}$ & ${ }^{3} \mathrm{P}$ & 5,6726 & 1,6082 & 1,56805 \\
$\mathrm{~N}$ & ${ }^{4} \mathrm{~S}$ & 6,6652 & 1,9236 & 1,9170 \\
$\mathrm{O}$ & ${ }^{3} \mathrm{P}$ & 7,6580 & 2,2461 & 2,22625 \\
$\mathrm{~F}$ & ${ }^{2} \mathrm{P}$ & 8,6501 & 2,5639 & 2,54985 \\
\hline
\end{tabular}

Tab. 3. Parameterwerte nach RansiL ${ }^{5}$ für den Ansatz II.

In Tab. 4 sind die Parameterwerte und die Energie für den Ansatz III angegeben. Auch diese Werte entstanden aus einer Interpolation, nachdem die optimalen Werte von $E$ und $k$ für $\beta=0,22 ; 0,24$; ...; 0,32 bestimmt waren ${ }^{6}$.

Die Unterschiede zwischen den theoretisch berechneten Energiewerten $E(\mathrm{I}), E(\mathrm{II})$ und $E(\mathrm{III})$ sind gering und klein gegen die Differenz zwischen diesen drei Werten und der experimentell bestimmten Energie. Das zeigt die Abb. 1, in der die drei mit I, II, III bezeichneten Striche die prozentuale Genauigkeit der theoretisch bestimmten Energie für die Ansätze I, II, III angeben. Die Abnahme von knapp $99,4 \%$ bei $\mathrm{B}, \mathrm{C}, \mathrm{N}$ nach $99,2 \%$ bei $\mathrm{Li}$ und $99,1 \%$ bei $\mathrm{F}$ hat ihren Grund in der in den Ansätzen I, II, III nicht berücksichtigten Elektronenkorrelation. Bei $\mathrm{B}$ gibt es nur ein p-Elektron, und in den Grundzuständen von $\mathrm{C}$ und $\mathrm{N}$ sind die beteiligten $\mathrm{p}$-Zustände verschiedene räumliche Zustände; erst von 0 an sind p-Zustände doppelt besetzt. Damit sollte die Elektronenkorrelation für $\mathrm{B}, \mathrm{C}, \mathrm{N}$ eine relativ geringere Bedeutung haben als für die anderen Atome und

\begin{tabular}{|c|c|c|c|c|c|c|c|c|}
\hline & & $k$ & $\beta$ & $\gamma$ & $k \beta$ & $k \gamma$ & $-E(\mathrm{H})$ & $-E(\operatorname{Exp})$. \\
\hline $\mathrm{Li}$ & ${ }^{2} \mathrm{~S}$ & 2,6864 & 0,2373 & - & 0,637 & - & 7,4179 & 7,4785 \\
\hline $\mathrm{Be}$ & ${ }^{1} \mathrm{~S}$ & 3,6845 & 0,2597 & - & 1,957 & - & 14,5567 & 14,6692 \\
\hline B & ${ }^{2} \mathrm{P}$ & 4,6791 & 0,2755 & 0,2586 & 1,289 & 1,210 & 24,4983 & 24,6578 \\
\hline $\mathrm{C}$ & $\begin{array}{l}{ }^{1} \mathrm{~S} \\
{ }^{1} \mathrm{D} \\
{ }^{3} \mathrm{P}\end{array}$ & $\begin{array}{l}5,6733 \\
5,6728 \\
5,6722\end{array}$ & $\begin{array}{c}0,2862 \\
0,2847 \\
0,2837\end{array}$ & $\begin{array}{l}0,2621 \\
0,2707 \\
0,2764\end{array}$ & $\begin{array}{l}1,623 \\
1,615 \\
1,609\end{array}$ & $\begin{array}{l}1,487 \\
1,535 \\
1,567\end{array}$ & $\begin{array}{l}37,4613 \\
37,5570 \\
37,6224\end{array}$ & $\begin{array}{l}37,7589 \\
37,8111 \\
37,8576\end{array}$ \\
\hline $\mathrm{N}$ & $\begin{array}{l}{ }^{2} \mathrm{P} \\
{ }^{2} \mathrm{D} \\
{ }^{4} \mathrm{~S}\end{array}$ & $\begin{array}{l}6,6656 \\
6,6654 \\
6,6650\end{array}$ & $\begin{array}{c}0,2903 \\
0,2896 \\
0,2886\end{array}$ & $\begin{array}{l}0,2802 \\
0,2832 \\
0,2877\end{array}$ & $\begin{array}{l}1,935 \\
1,931 \\
1,924\end{array}$ & $\begin{array}{l}1,868 \\
1,888 \\
1,917\end{array}$ & $\begin{array}{l}54,0692 \\
54,1484 \\
54,2688\end{array}$ & $\begin{array}{l}54,4819 \\
54,5258 \\
54,6133\end{array}$ \\
\hline $\mathrm{O}$ & $\begin{array}{l}{ }^{1 \mathrm{~S}} \\
{ }^{1} \mathrm{D} \\
{ }^{3} \mathrm{P}\end{array}$ & $\begin{array}{l}7,6577 \\
7,6576 \\
7,6574\end{array}$ & $\begin{array}{l}0,2947 \\
0,2939 \\
0,2934\end{array}$ & $\begin{array}{l}0,2862 \\
0,2890 \\
0,2908\end{array}$ & $\begin{array}{l}2,257 \\
2,250 \\
2,247\end{array}$ & $\begin{array}{l}2,192 \\
2,213 \\
2,227\end{array}$ & $\begin{array}{l}74,3074 \\
74,4468 \\
74,5404\end{array}$ & $\begin{array}{l}74,9592 \\
75,0408 \\
75,1131\end{array}$ \\
\hline $\mathrm{F}$ & ${ }^{2} \mathrm{P}$ & 8,6502 & 0,2966 & 0,2947 & 2,565 & 2,549 & 98,9423 & 99,8179 \\
\hline $\mathrm{Ne}$ & ${ }^{1} \mathrm{~S}$ & 9,6437 & 0,2988 & 0,2983 & 2,881 & 2,876 & 127,8124 & - \\
\hline
\end{tabular}

Tab. 2. Parameterwerte und Energien für den Ansatz II. $k$ in reziproken Bohrschen Radien, Energie $E$ in doppelte RydBerg.

4 B. Kockel u. S. Rühlemann, Phys. Bl. 16, 174 [1960].

5 B. J. Ransil, Rev. Mod. Phys. 32, 239 [1960].

6 An einer Stelle $\left(\mathrm{N}^{4} \mathrm{~S}\right)$ ist $|E(\mathrm{III})|>|E(\mathrm{II})|$, was sich nicht ergeben dürfte. Der Grund dafür ist, daß für die Werte in Tab. 4 ein genaueres Interpolationsverfahren (über vier $\beta$-Werte) verwendet wurde. 


\begin{tabular}{|c|c|c|c|c|c|c|}
\hline & & $k$ & $\beta$ & $k \beta$ & $-E(\mathrm{III})$ & $-E(\operatorname{Exp})$. \\
\hline $\mathrm{Li}$ & ${ }^{2} \mathrm{~S}$ & 2,6865 & 0,2372 & 0,6372 & 7,4179 & 7,4785 \\
\hline $\mathrm{Be}$ & ${ }^{1} \mathrm{~S}$ & 3,6845 & 0,2597 & 0,9568 & 14,5567 & 14,6692 \\
\hline B & $2 \mathrm{P}$ & 4,6800 & 0,2711 & 1,2687 & 24,4972 & 24,6578 \\
\hline C & ${ }^{3} \mathrm{P}$ & 5,6734 & 0,2806 & 1,5920 & 37,6218 & 37,8576 \\
\hline $\mathrm{N}$ & ${ }^{4} \mathrm{~S}$ & 6,6653 & 0,2881 & 1,9203 & 54,2689 & 54,6133 \\
\hline O & ${ }^{3} \mathrm{P}$ & 7,6585 & 0,2918 & 2,2348 & 74,5402 & 75,1131 \\
\hline F & ${ }^{2} \mathrm{P}$ & 8,6506 & 0,2954 & 2,5551 & 98,9420 & 99,8179 \\
\hline $\mathrm{Ne}$ & ${ }^{1} \mathrm{~S}$ & 9,6418 & 0,2986 & 2,8795 & 127,8122 & - \\
\hline
\end{tabular}

Tab. 4. Parameter und Energien für den Ansatz III.

deshalb eine die Korrelation nicht berücksichtigende Zustandsfunktion für $\mathrm{B}, \mathrm{C}, \mathrm{N}$ zu einem relativ besseren Wert für die Energie führen.

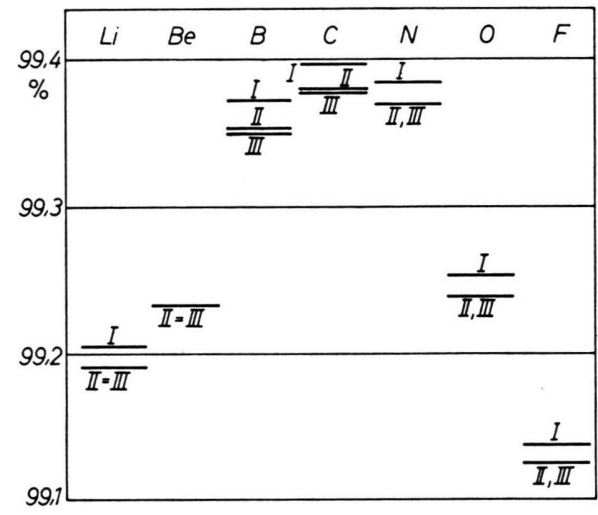

Abb. 1. Die prozentische Genauigkeit der theoretisch berechneten Energie der Grundzustände der Atome Lithium bis Neon für die Ansätze I bis III. II=III heißt: Die Zustände nach II und III unterscheiden sich nicht. II, III heißt: Innerhalb der Strichdicke ist die Genauigkeit gleich.

\section{Zustandsfunktionen für einfach positive Ionen}

Für den Grundzustand einfach positiver Ionen ist zu erwarten, daß seine Elektronenkonfiguration, sein Drehimpuls und der Spin gleich denen des im periodischen System vorangehenden neutralen Atoms sind. Für die Ionen $\mathrm{Li}^{+}$bis $\mathrm{Ne}^{+}$entsteht so die folgende Tabelle und durch Vergleich mit den Energiewerten der neutralen Atome die Tab. 6. Man sieht, daß die experimentellen Ionisierungsenergien nur schlecht wiedergegeben werden - der Fehler steigt bis zum F auf $40 \%$. Der Grund dafür ist natürlich, $\mathrm{da} ß$ die berechneten Ionisierungsenergien als Differenzen zweier beinahe gleich großer Zahlen entstehen. Bei dem 4-konstantigen Ansatz I ergeben sich die gleichen großen Abweichungen - man ver-

7 B. KockeL, Z. Naturforschg. 14 a, 1088 [1959].

\begin{tabular}{|l|c|c|c|c|r|}
\hline & & $k$ & $\beta$ & $k \beta$ & $-E$ (III) \\
\hline $\mathrm{Li}^{+}$ & ${ }^{1} \mathrm{~S}$ & 2,6875 & - & - & 7,2227 \\
$\mathrm{Be}^{+}$ & ${ }^{2} \mathrm{~S}$ & 3,6818 & 0,2950 & 1,086 & 14,2584 \\
$\mathrm{~B}^{+}$ & ${ }^{1} \mathrm{~S}$ & 4,6761 & 0,2987 & 1,397 & 24,2136 \\
$\mathrm{C}^{+}$ & ${ }^{2} \mathrm{P}$ & 5,6667 & 0,3048 & 1,727 & 37,2520 \\
$\mathrm{~N}^{+}$ & ${ }^{3} \mathrm{P}$ & 6,6571 & 0,3095 & 2,060 & 53,8099 \\
$\mathrm{O}^{+}$ & ${ }^{4} \mathrm{~S}$ & 7,6467 & 0,3131 & 2,394 & 74,2270 \\
$\mathrm{~F}^{+}$ & ${ }^{3} \mathrm{P}$ & 8,6390 & 0,3140 & 2,713 & 98,5519 \\
$\mathrm{Ne}^{+}$ & ${ }^{2} \mathrm{P}$ & 9,6311 & 0,3154 & 3,037 & 127,3431 \\
\hline
\end{tabular}

Tab. 5. Parameter und Energien für einfach positive Ionen.

gleiche dazu z. B. die Abb. 7 in der unten zitierten Arbeit ${ }^{7}$.

Die Zahlen für $k \beta$ in Tab. 5 sind sofort verständlich. Sie sind größer als beim neutralen Atom, weil jetzt ein Elektron weniger in der L-Schale vorhanden ist; denn dadurch ist der Beitrag der CoulombAbstoßung der Elektronen zur Energie verringert, und die Elektronen können näher an den Kern heranrücken. Im ersten Augenblick weniger verständlich ist die kleine Abnahme der Abfallexponenten für die ls-Elektronen bei den Ionen $\mathrm{Be}^{+}$bis $\mathrm{Ne}^{+}$. Der Grund dafür ist die beim positiven Ion geringere Herandrängung der K-Elektronen durch die Außenelektronen an den Kern. Daß ein solcher Effekt vorhanden ist, weiß man z. B. aus Berechnungen des $\mathrm{H}^{-}$-Ions und des He-Atoms mit radialer Korrelation, wobei sich bei dem Ansatz

$$
\psi=e^{-k r_{1}-k^{\prime} r_{2}}+e^{-k^{\prime} r_{1}-k r_{2}}
$$

für $\mathrm{H}^{-}$

$$
\begin{aligned}
\psi & =e^{-1,039 r-0,283 r^{\prime}}+e^{-0,283 r^{\prime}-1,039 r} ; \\
-E & =0,5133 \mathrm{DRy}
\end{aligned}
$$

für $\mathrm{He}$

$$
\begin{aligned}
\psi & =e^{-2,183 r-1,189 r^{\prime}}+e^{-1,189 r-2,183 r^{\prime}} ; \\
-E & =2,8757 \mathrm{DRy}
\end{aligned}
$$

ergibt (Normierungsfaktoren sind weggelassen). Der größere Exponent ist größer als die Kernladung, und das bedeutet eine Konzentration für die 


\begin{tabular}{|l|c|c|c|c|c|c|c|c|}
\hline $\begin{array}{l}\text { Erste Ioni- } \\
\text { sierungsenergie }\end{array}$ & $\mathrm{Li}$ & $\mathrm{Be}$ & $\mathrm{B}$ & $\mathrm{C}$ & $\mathrm{N}$ & $\mathrm{O}$ & $\mathrm{F}$ & $\mathrm{Ne}$ \\
\hline für Ansatz III & 0,1952 & 0,2983 & 0,2836 & 0,3698 & 0,4590 & 0,3132 & 0,3901 & 0,4691 \\
experimentell & 0,1982 & 0,3426 & 0,3046 & 0,4141 & 0,5347 & 0,5005 & 0,6404 & 0,7185 \\
\hline
\end{tabular}

Tab. 6. Erste Ionisierungsenergie der Elemente Lithium bis Neon. Alle Werte in doppelten RydBERG $=27,2 \mathrm{eV}$.

der zugehörigen Exponentialfunktion entsprechende Elektronendichte um den Kern, die stärker ist als bei den Einelektronensystemen $\mathrm{H}$ und $\mathrm{He}^{-}$.

\section{Zustandsfunktionen für einfach negative Ionen}

Ein Versuch, für die negativen Ionen dieselbe Elektronensituation anzunehmen wie für das im periodischen System folgende neutrale Atom und zugleich bei den einfachen Zustandsfunktionen der Ansätze I bis III zu bleiben, scheitert. Die Energiewerte werden höher (dem Betrag nach kleiner) als die Energiewerte der Atome. Deshalb hat es keinen Sinn, für die auf diese Weise berechneten Ionen die Parameterwerte anzugeben. In Tab. 7 sind deshalb

\begin{tabular}{|l|l|l|l|l|l|}
\hline & & $-E(\mathrm{III})$ & & & $-E(\mathrm{III})$ \\
\hline $\mathrm{H}^{-}$ & ${ }^{1} \mathrm{~S}$ & 0,4727 & $\mathrm{H}$ & ${ }^{2} \mathrm{~S}$ & 0,5000 \\
$\mathrm{He}^{-}$ & ${ }^{2} \mathrm{~S}$ & $2,8477^{8}$ & $\mathrm{He}$ & ${ }^{1} \mathrm{~S}$ & 2,8477 \\
$\mathrm{Li}^{-}$ & ${ }^{1} \mathrm{~S}$ & 7,4108 & $\mathrm{Li}$ & ${ }^{2} \mathrm{~S}$ & 7,4179 \\
$\mathrm{Be}^{-}$ & ${ }^{2} \mathrm{P}$ & 14,4982 & $\mathrm{Be}$ & ${ }^{1} \mathrm{~S}$ & 14,5567 \\
$\mathrm{~B}^{-}$ & ${ }^{3} \mathrm{P}$ & 24,4367 & $\mathrm{~B}$ & ${ }^{2} \mathrm{P}$ & 24,4972 \\
$\mathrm{C}^{-}$ & ${ }^{4} \mathrm{~S}$ & 37,5630 & $\mathrm{C}$ & ${ }^{3} \mathrm{P}$ & 37,6218 \\
$\mathrm{~N}^{-}$ & ${ }^{3} \mathrm{P}$ & 54,0290 & $\mathrm{~N}$ & ${ }^{4} \mathrm{~S}$ & 54,2689 \\
$\mathrm{O}^{-}$ & ${ }^{2} \mathrm{P}$ & 74,2904 & $\mathrm{O}$ & ${ }^{3} \mathrm{P}$ & 74,5402 \\
$\mathrm{~F}^{-}$ & ${ }^{1} \mathrm{~S}$ & 98,6801 & $\mathrm{~F}$ & ${ }^{2} \mathrm{P}$ & 98,9420 \\
\hline
\end{tabular}

Tab. 7. Energie negativer Ionen für die Zustandsfunktionen (1) und zum Vergleich die Energie der neutralen Atome.

als Beleg für diese Aussage nur die Energie der Ionen und zum Vergleich die der Atome angegeben. Die zur Berechnung benutzten Zustandsfunktionen sind dabei also die Stater-Determinanten oder Linearkombinationen von SLATER-Determinanten, die auch für die neutralen Atome gleicher Elektronenzahl benutzt werden, $z$. B.

$$
\begin{aligned}
& \mathrm{H}^{-1} \mathrm{~S} \quad \psi=\operatorname{det} \mid 1 \mathrm{~s} \chi_{+} \text {ls } \chi_{-} \mid \text {, } \\
& \mathrm{He}^{-2} \mathrm{~S} \quad \psi=\operatorname{det}\left|\operatorname{ls} \chi_{+} 1 \mathrm{~s} \chi_{-} 2 \mathrm{~s} \chi_{+}\right| \text {, } \\
& \mathrm{F}^{-1} \mathrm{~S} \quad \psi=\operatorname{det} \mid 1 \mathrm{~s} \chi_{+} 1 \mathrm{~s} \chi_{-} 2 \mathrm{~s} \chi_{+} 2 \mathrm{~s} \chi_{-} \\
& 2 \mathrm{p}^{(1)} \chi_{+} 2 \mathrm{p}^{(1)} \chi_{-} 2 \mathrm{p}^{(0)} \chi_{+} 2 \mathrm{p}^{(0)} \chi_{-} \\
& 2 \mathrm{p}^{(-1)} \chi_{+} 2 \mathrm{p}^{(-1)} \chi_{-} \mid \text {, }
\end{aligned}
$$

${ }^{8} \mathrm{Bei} \mathrm{He}^{-}$hat der Energiemittelwert erst für $k \beta=0$, also nicht gebundenes drittes Elektron, ein Minimum. mit den 1s-, 2s-, 2p-Funktionen des Ansatzes III und den Spinfunktionen $\chi_{+}$und $\chi_{-}$. Für $\mathrm{Li}^{+}, \mathrm{Li}$ und $\mathrm{Li}^{-}$findet man auch eine Berechnung in einer Arbeit von Roothana, Sachs und Weiss ${ }^{9}$. Sie verwenden Linearkombinationen mehrerer Exponentialfunkitonen, von denen jede noch mit einer Potenzreihe in $r$ multipliziert ist, also eine Funktion mit großer Konstantenzahl. Für die beiden 2s-Elektronen des $\mathrm{Li}^{-}$-Ions setzen sie ebenfalls die gleiche Ortsfunktion an und erhalten auch für $\mathrm{Li}^{-}$eine geringere Bindungsenergie als für $\mathrm{Li}$ :

\begin{tabular}{|l|c|c|c|c|}
\hline & & $\mathrm{Li}^{+}$ & $\mathrm{Li}$ & $\mathrm{Li}^{-}$ \\
\hline $\begin{array}{l}\text { RoOTHAAN, SACHS, } \\
\text { WeisS }\end{array}$ & $-E$ & 7,2364 & 7,4327 & 7,4282 \\
\hline
\end{tabular}

Die auf Grund von Tab. 7 zu ziehende Folgerung ist natürlich die, daß die negativen Ionen in ihrem Aufbau nicht den Atomen derselben Elektronenzahl gleichen. Eines der Elektronen der negativen Ionen ist viel „weiter draußen“ eingebaut, eine der in die Zustandsfunktion eingehenden einzelnen Elektronenfunktionen muß also einen wesentlich kleineren $\mathrm{Ab}$ fallexponenten haben. Berücksichtigt man das, so werden sich alle negativen Ionen der Tab. 7 als existent erweisen - mit Ausnahme von $\mathrm{He}^{-}$, bei dem der Ansatz (1) ja schon das dritte Elektron in einen neuen Zustand an den Grundzustand (1s) ${ }^{2}$ des neutralen Heliums anlagert.

Für die Ionen $\mathrm{H}^{-}$und $\mathrm{Li}^{-}$soll diese Aussage bewiesen werden an der Berechnung der Zustände $1 \mathrm{~s} 1 \mathrm{~s}^{\prime}{ }^{1} \mathrm{~S}$ für $\mathrm{H}^{-},(1 \mathrm{~s})^{2} 2 \mathrm{~s} 2 \mathrm{~s}^{\prime}{ }^{1} \mathrm{~S}$ für $\mathrm{Li}^{-}$. Die Eigenfunktionen sind also

$$
\begin{aligned}
& \mathrm{H}^{-}: \quad \psi=\operatorname{det}\left|1 \mathrm{~s} \chi_{+} 1 \mathrm{~s}^{\prime} \chi_{-}\right|-\operatorname{det}\left|1 \mathrm{~s} \chi_{-} 1 \mathrm{~s}^{\prime} \chi_{+}\right| \\
& \mathrm{Li}^{-}: \quad \psi=\operatorname{det} \mid 1 \mathrm{~s} \chi_{+} \text {ls } \chi_{-} 2 \mathrm{~s} \chi_{+} 2 \mathrm{~s}^{\prime} \chi_{-} \mid \\
& -\operatorname{det}\left|1 \mathrm{~s} \chi_{+} 1 \mathrm{~s} \chi_{-} 2 \mathrm{~s} \chi_{-} 2 \mathrm{~s}^{\prime} \chi_{+}\right|
\end{aligned}
$$

9 C. C. J. RoothaAn, L. M. SAchs u. A. W. Weiss, Phys. Rev. 32, 186 [1960]. 
Dabei erhält man für $\mathrm{H}^{-}$die schon im vorigen $\mathrm{Ab}$ schnitt mitgeteilten Resultate

$\mathrm{H}^{-}$:

$$
\begin{gathered}
k=1,039-E=0,5133 \mathrm{DRy}, \quad k^{\prime}=0,283 \\
\text { Elektronenaffinität } 0,0133 \mathrm{DRy}=0,36 \mathrm{eV} .
\end{gathered}
$$

Die Elektronenaffinität ergibt sich also bei dieser einfachen Zustandsfunktion schon zu 50\% der besten bekannten, mit viel ausführlicheren Zustandsfunktionen berechneten Werte.

In diesen besseren Zustandsfunktionen, die in der Form

$\psi=e^{-k\left(r_{1}+r_{2}\right)} \cdot$ Potenzreihe in $\left(r_{1}+r_{2}\right),\left(r_{1}-r_{2}\right)^{2}$,

$$
r_{12} \cdot\left\{\chi_{+}(1) \chi_{-}(2)-\chi_{-}(1) \chi_{+}(2)\right\}
$$

mit $r_{12}=$ Elektronenabstand angesetzt werden, ist die Aussage, daß ein Elektron sich „weit draußen“ aufhält, in den $\left(r_{1}-r_{2}\right)^{2}$-Gliedern enthalten, die ja bei einer Entwicklung von (2) gemäß

$$
\begin{aligned}
\psi= & 2 e^{-\left(k+k^{\prime}\right) / 2\left(r_{1}+r_{2}\right)} \\
& \cdot\left\{1+\frac{\left(k-k^{\prime}\right)^{2}}{8}\left(r_{1}-r_{2}\right)^{2}+\frac{\left(k-k^{\prime}\right)^{4}}{384}\left(r_{1}-r_{2}\right)^{4} \ldots\{\right.
\end{aligned}
$$

auch sofort auftreten.

Für $\mathrm{Li}^{-}$ergibt sich

$$
\begin{aligned}
k=0,2867, \beta & =0,2449, k \beta=0,6579, \\
\beta^{\prime} & =0,0932, k \beta^{\prime}=0,2504,
\end{aligned}
$$

also eine Elektronenaffinität von 0,0033 DRy= $0,09 \mathrm{eV}$. Dieses Resultat ist viel schlechter als andere Ergebnisse ${ }^{10}$. Es reicht trotzdem aus als Beleg für die Aussage, daß eine der beteiligten Einelektronenfunktionen ein weit vom Kern entferntes Elektron bedeuten muß.

Zur Veranschaulichung stellen die Abb. 2 und 3 die radiale Elektronenverteilung für beide Elektronen im $\mathrm{H}^{-}$-Ion und für die Außenelektronen im $\mathrm{Li}^{-}$Ion dar, zeigen also $4 \pi r^{2} \psi_{1 \mathrm{~s}}{ }^{2}, 4 \pi r^{2} \psi_{1 \mathrm{~s}^{\prime}}{ }^{2} \mathrm{bzw}$. $4 \pi r^{2} \psi_{2 s^{2}}{ }^{2}, 4 \pi r^{2} \psi_{2 s^{\prime}}{ }^{2}$ in Abhängigkeit von $r$.

\section{Terme des C-Atoms}

Für die Berechnung von Kohlenstoffverbindungen ist es schließlich noch nützlich, die Lage der drei tiefsten geraden Terme $\left\{1 \mathrm{~s}^{2}, 2 \mathrm{~s}^{2}, 2 \mathrm{p}^{2}{ }^{3} \mathrm{P},{ }^{1} \mathrm{D},{ }^{1} \mathrm{~S}\right\}$ und die des tiefsten ungeraden Terms $1 \mathrm{~s}^{2}, 2 \mathrm{~s}, 2 \mathrm{p}^{35} \mathrm{~S}$ zu kennen. Die Tab. 8 und das linke Drittel der Abb. 4 zeigen das Ergebnis, wenn man nur die $\mathrm{Zu}$ -

10 Landolt-Börnstein, Zahlenwerte und Funktionen 1950, 1. Band, 1. Teil, S. 213.

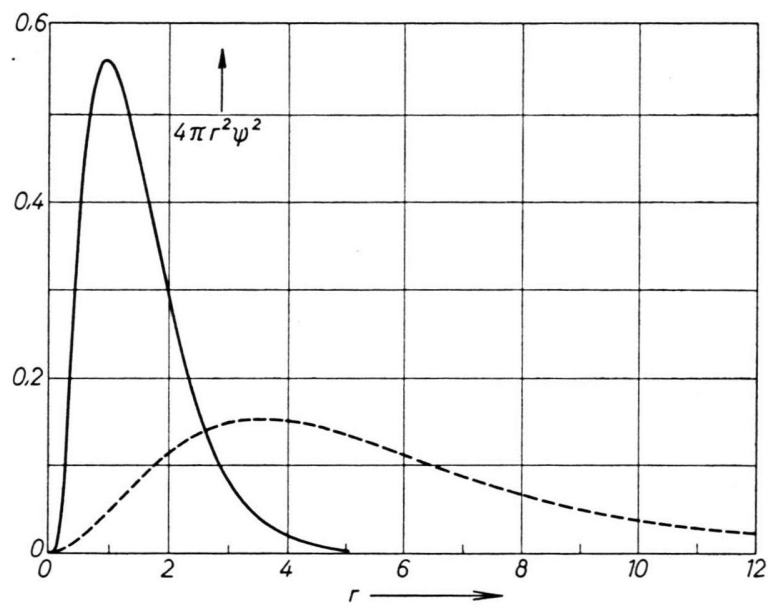

Abb. 2. Radiale Elektronenverteilung für beide Elektronen des $\mathrm{H}^{-}$-Ions mit den Zustandsfunktionen $e^{-k r}, e^{-k^{\prime} r}$; $k=1,039 ; k^{\prime}=0,283$.

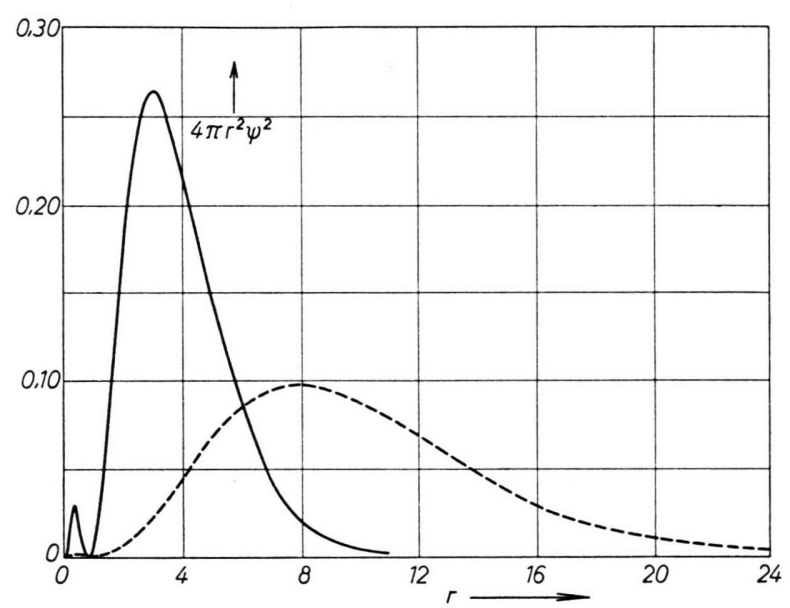

Abb. 3. Radiale Elektronenverteilung der beiden Außenelektronen im $\mathrm{Li}^{-}$-Ion. Zustandsfunktionen

$\left\llcorner r e^{-k \beta r}, e^{-k r} \downarrow ; \leftarrow L^{r} e^{-k \beta^{\prime} r}, e^{-k r}\right] ; k=2,6867 ;$
$k \beta=0,6579 ; k \beta^{\prime}=0,2504$.

\begin{tabular}{|c|c|c|c|c|c|}
\hline $\begin{array}{c}\text { Kon- } \\
\text { figuration }\end{array}$ & Term & $k$ & $\beta$ & $k \beta$ & $-E(\mathrm{III})$ \\
\hline $1 s^{2} 2 s^{2} 2 p^{2}$ & ${ }^{1} \mathrm{~S}$ & 5,6760 & 0,2763 & 1,568 & 37,4552 \\
\hline $1 s^{2} 2 s^{2} 2 p^{2}$ & ${ }^{1} \mathrm{D}$ & 5,6744 & 0,2789 & 1,583 & 37,5549 \\
\hline $1 \mathrm{~s}^{2} 2 \mathrm{~s}^{2} 2 \mathrm{p}^{2}$ & ${ }^{3} \mathrm{P}$ & 5,6734 & 0,2806 & 1,592 & 37,6218 \\
\hline $1 s^{2} 2 s 2 p^{3}$ & ${ }^{5} \mathrm{~S}$ & 5,6698 & 0,2837 & 1,609 & 37,5227 \\
\hline
\end{tabular}

$$
k \beta=0,6579 ; k \beta^{\prime}=0,2504 \text {. }
$$

standsfunktionen des Ansatzes III verwendet. Beim Vergleich mit experimentellen Energiewerten (letztes Drittel der Abb. 4) zeigt sich, daß die Genauigkeit der theoretischen Termwerte $99,2-99,5 \%$ ist, daß die relative Lage der geraden Terme zueinan-

Tab. 8. Einige Terme des C-Atoms. 


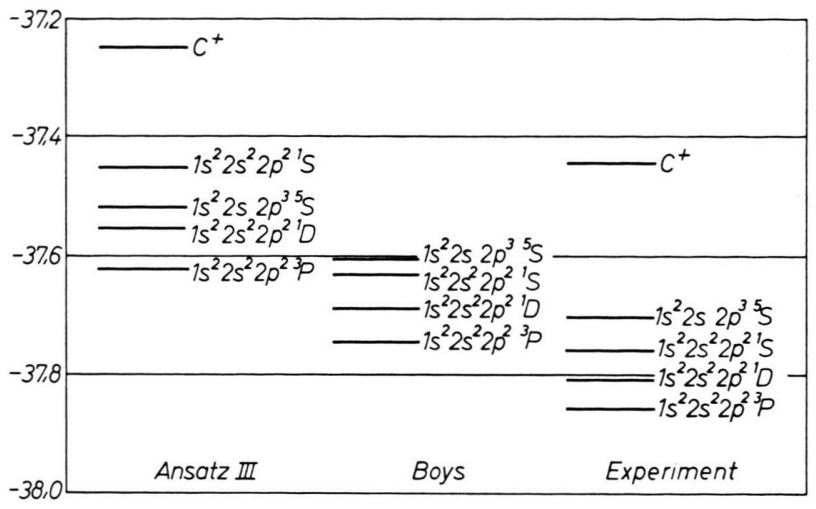

Abb. 4. Terme des C-Atoms nach Tab. 8, Boys ${ }^{11}$ und dem Experiment. der nur grob qualitativ mit dem Experiment übereinstimmt und daß der ${ }^{5} \mathrm{~S}$-Term zwischen dem ${ }^{1} \mathrm{~S}$ und dem ${ }^{1} \mathrm{D}$-Term statt über beiden liegt. Eine Verbesserung dieser theoretischen Ergebnise ist nur durch wesentlich ausführlichere Rechnungen möglich, in denen mindestens noch alle Zustände mit einem Elektron in der M-Schale und ihre Konfigurationswechselwirkung mit den Funktionen nach Ansatz III zu berücksichtigen wären ${ }^{12}$. Damit würde man aber zu Zustandsfunktionen gelangen, die für Molekülberechnungen zu unhandlich sind. Dasselbe gilt für die an sich viel besseren Zustandsfunktionen von Boys ${ }^{11}$, dessen Termwerte im mittleren Drittel von Abb. 4 eingetragen sind.

Terme nur mit den $(1 s)^{2}(2 p)^{4}$-Termen führt schon zur richtigen Reihenfolge ${ }^{5} \mathrm{~S},{ }^{1} \mathrm{~S},{ }^{1} \mathrm{D},{ }^{3} \mathrm{P}$.

\title{
Ionen-Molekülreaktionen in Paraffinen, Olefinen und Azetylen
}

\author{
Von R. Fuchs * \\ Mitteilung aus der Physikalisch-Technischen Bundesanstalt \\ (Z. Naturforschg. 16 a, 1026-1037 [1961] ; eingegangen am 15. Juni 1961)
}

\begin{abstract}
Bei den Kohlenwasserstoffen $\mathrm{CH}_{4}, \mathrm{C}_{2} \mathrm{H}_{6}, \mathrm{C}_{3} \mathrm{H}_{8}, \mathrm{C}_{2} \mathrm{H}_{4}, \mathrm{C}_{3} \mathrm{H}_{6}, 1-\mathrm{C}_{4} \mathrm{H}_{8}$, iso $-\mathrm{C}_{4} \mathrm{H}_{8}$, trans $-\mathrm{C}_{4} \mathrm{H}_{8}$ und $\mathrm{C}_{2} \mathrm{H}_{2}$ wurden die im Ionisierungsraum eines Massenspektrometers durch Ionen-Molekülreaktionen gebildeten Sekundärionen untersucht. Die Bildungsreaktionen wurden, soweit als möglich, mit Hilfe von Auftrittspotential-Messungen ermittelt und ihre Wirkungsquerschnitte bestimmt. Bei Propen und den Butenen läßt ein Vergleich der Häufigkeiten der durch Reaktionen von Molekülionen gebildeten Sekundärionen mit primären Massenspektren von Verbindungen der Zusammensetzung $\mathrm{C}_{6} \mathrm{H}_{12}$ bzw. $\mathrm{C}_{8} \mathrm{H}_{16}$ Schlüsse auf die Strukturen der bei den Reaktionen gebildeten Übergangskomplexe zu. Bei den Paraffinen geht die gesamte Sekundärionenbildung mit zunehmendem Molekulargewicht stark zurück. Sie lag bei den Butanen unterhalb der Nachweisgrenze. Eine Zunahme ist dagegen in der Reihe $\mathrm{C}_{2} \mathrm{H}_{6}, \mathrm{C}_{2} \mathrm{H}_{4}, \mathrm{C}_{2} \mathrm{H}_{2}$ festzustellen. Dies deutet darauf hin, daß außer der Polarisierbarkeit auch die Struktur der Moleküle die Reaktionsquerschnitte wesentlich beeinflußt.
\end{abstract}

Ionen-Molekülreaktionen in der Gasphase, die sich in Massenspektrometerionenquellen schon bei mäßig erhöhtem Gasdruck durch das Auftreten von Sekundärionen bemerkbar machen, haben, nicht zuletzt auch im Hinblick auf die Rolle, die sie bei den primären Vorgängen strahlenchemischer Prozesse spielen, in der vergangenen Zeit vermehrtes Interesse gefunden: Grundsätzliche Fragen des Reaktions-

* Braunschweig.

1 D. P. Stevenson u. D. O. Schissler, a) J. Chem. Phys. 23, 1353 [1955]. b) J. Chem. Phys. 29, 282 [1958].

2 D. O. Schissler u. D. P. Stevenson, J. Chem. Phys. 24, 926 [1956].

3 G. Gioumousis u. D. P. Stevenson, J. Chem. Phys. 29, 294 [1958].

4 F. H. Field, J. L. Franklin u. F. W. Lampe, a) J. Amer. mechanismus wurden besonders von STEvenson und Mitarbeitern ${ }^{1-3}$ an Reaktionen einfacher Moleküle sowohl experimentell als auch theoretisch untersucht. Messungen an verschiedenen Kohlenwasserstoffen wurden von Field, Franklin und Lampe ${ }^{4,5}$ sowie von Hamill und Mitarbeitern ${ }^{6,7}$ ausgeführt. Dabei wird jedoch nur bei einigen Gasen ein vollständigerer Überblick über die Sekundärionenbildung gege-

Chem. Soc. 79, 2419 [1957] ; b) J. Amer. Chem. Soc. 79, 2665 [1957].

5 F. H. Field u. F. W. Lampe, J. Amer. Chem. Soc. a) 80, 5583 [1958] ; b) 80, 5587 [1958] ; c) 81, 3242 [1959].

6 R. Barker, W. H. Hamill u. R. R. Williams jr., J. Phys. Chem. 63, 825 [1959].

7 R. F. Pottie, R. Barker u. W. H. Hamill, Rad. Res. 10, 664 [1959]. 